\title{
Identification of a noncoding RNA-mediated gene pair-based regulatory module in Alzheimer's disease
}

\author{
LIN YU, SHI QIAN and SUN WEI
}

Department of Neurology, The First Affiliated Hospital of Harbin Medical University, Harbin, Heilongjiang 150081, P.R. China

Received December 12, 2017; Accepted May 22, 2018

DOI: $10.3892 / \mathrm{mmr} .2018 .9190$

\begin{abstract}
Alzheimer's disease (AD) is the most common type of neurological disorder that results from brain cell death; however, not all brain regions are simultaneously affected to the same extent. Despite single biomarkers for AD having been determined on a genome-wide scale, the differential co-expression in gene pairs between regions and interactions with other types of cellular molecules, particularly non-coding (nc)RNAs, are often overlooked in studies investigating the underlying mechanisms associated with AD. In the present study, based on 1,548 samples obtained from a cohort of 90 patients with AD spanning 19 brain regions, a gene-pair based method was established for the classification of 19 brain regions into seven different groups, including marked disparate groupings of six single regions and a cluster of another 13 regions as revealed by principal component analysis (PCA). To further investigate the different underlying mechanisms associated with each group, five highly interconnected functional modules of the protein-protein interaction network were demonstrated to characterize the seven region groups containing six single groups and 13 clustered regions based on 4,731 gene-pairs. Genes in two of the functional modules exhibited a strong association with pathways associated with the nervous system, including cholinergic synapses, circadian entrainment and dopaminergic synapses. Notably, following integration of these two modules with a ncRNA-mediated network, one module demonstrated a strong association with micro (mi)RNAs, which were revealed to interact with numerous long non-coding (lnc)RNAs associated with AD, such as metastasis associated lung adenocarcinoma transcript 1 and taurine upregulated 1 . This suggested that
\end{abstract}

Correspondence to: Dr Sun Wei, Department of Neurology, The First Affiliated Hospital of Harbin Medical University, 23 Youzheng Street, Harbin, Heilongjiang 150081, P.R. China

E-mail: sunwei70398@163.com

Abbreviations: AD, Alzheimer's disease; lncRNA, long non-coding RNA; miRNA, microRNA; ncRNA, non-coding RNA; PCA, principal component analysis; ceRNA, competing endogenous RNA

Key words: Alzheimer's disease, ncRNAs, ceRNA, gene-pair-based method, network-based modules
mRNAs and lncRNAs may represent competing endogenous RNAs for binding with miRNAs. Thus, these results indicated that the ncRNA-mediated gene regulatory module detected by the established gene pair-based method may further the understanding of underlying mechanisms associated with AD as well as aid the development of novel therapeutic strategies for the treatment of patients with AD.

\section{Introduction}

Alzheimer's disease (AD) is the most common form of neurological disorder, presenting symptoms of irreversible and progressive memory loss as well as cognitive decline as a result of the death of brain cells (1). In 2017, a total of 5.5 million people suffered from AD in the USA, of which 5.3 million were aged $\geq 65$ years old (2). However, not all brain regions associated with $\mathrm{AD}$ are simultaneously affected to the same extent during disease progression $(3,4)$. Therefore, it is of critical importance to determine the different underlying mechanisms affecting different brain regions during AD.

As high-throughput technologies have advanced, multi-level omics data are subsequently becoming widely available (5). Due to the increasing production of large-scale functional genomic datasets, single biomarkers for AD are now being determined on a genome-wide scale $(6,7)$. Cellular genes demonstrating a correlation in expression changes are likely to function as a gene pair that perform similar functions $(8,9)$. However, gene pairs can be present in biological networks that interact with numerous molecular regulators, such as micro (mi)RNAs and long non-coding (lnc)RNAs, which serve important roles in regulating transcription, post-transcriptional modification and translation as well as non-coding (nc)RNAs in humans (10-12). LncRNAs have numerous structural features that are similar to messenger (m)RNAs; for example, lncRNAs may recognize complementary sequences of mRNAs, and thus regulate mRNA processing (13). Whereas miRNAs negatively regulate gene expression via the targeting of mRNAs (14). However, interactions between miRNAs and mRNAs are not unidirectional, and mRNAs and lncRNAs (15) can function as competing endogenous (ce)RNAs for binding with miRNAs (16). For example, the cell division cycle $42 \mathrm{AD}$ associated gene and the ribonuclease $\mathrm{P}$ RNA component H1 lncRNA represent a compensatory mechanism in the early stage of AD pathogenesis (17). Furthermore, modularity has been considered to represent an important indicator for the identification of key molecules present in 
multi-level ncRNA-mediated gene regulatory networks in order to further the understanding of AD in different brain regions.

In the present study, based on 1,548 samples from a cohort of 90 patients with AD spanning 19 brain regions from Gene Expression Omnibus (GEO) database (database no. GSE84422) (3), a gene pair-based method was established for the classification of 19 brain regions into different groups according to exhibited symptoms associated with AD (Fig. 1). Principal component analysis (PCA) revealed that there were marked differences in the effect of AD on the amygdala, caudate nucleus, nucleus accumbens, precentral gyrus, putamen and temporal pole brain regions as well as a cluster of a further 13 brain regions disparately affected by AD. Five functional modules were identified to distinguish these regional groupings based on the most strongly associated gene-pairs revealed by PCA. Functional annotation of genes in two of the modules suggested a strong correlation with pathways associated with the nervous system, including cholinergic synapses, circadian entrainment and dopaminergic synapses. Notably, following integration of the two modules with interactions between miRNAs, lncRNAs and mRNAs, as revealed by RNA Association Interaction Database (RAID) v.2.0 analysis (18), one module demonstrated a close association with miRNAs, which, on the contrary, interacted with numerous lncRNAs associated with $\mathrm{AD}$, such as metastasis associated lung adenocarcinoma transcript 1 (MALAT1) and non-protein coding taurine upregulated 1 (TUG1), which suggested that mRNAs and lncRNAs may represent ceRNAs for binding with miRNAs. These results suggested that this classification-represented ncRNA-mediated gene regulatory module established by the gene-pair-based method used in the present study may further the understanding of the mechanisms underlying the pathogenesis of AD.

\section{Materials and methods}

Gene expression and RNA-RNA interactions. Gene expression data of 1,548 samples obtained from a cohort of 90 patients with AD spanning 19 brain regions generated by HG-U133A, HG-U133B and HG-U133_Plus_2 platforms were downloaded from the GEO database (database no. GSE84422) (3). The 19 brain regions included the amygdala, anterior cingulate, caudate nucleus, dorsolateral prefrontal cortex, frontal pole, hippocampus, inferior frontal gyrus, inferior temporal gyrus, middle temporal gyrus, nucleus accumbens, occipital visual cortex, parahippocampal gyrus, posterior cingulate cortex, precentral gyrus, prefrontal cortex, putamen, superior parietal lobule, superior temporal gyrus and temporal pole. Robust multi-array average-normalized microarray data were downloaded (19). Replicate genes were combined by taking the mean of their expression values. In order to minimize variance induced by technical variation, batch effects were removed using the R/Bioconductor package $s v a \mathrm{v}$ 3.24.4 (20), which is based on surrogate variable analysis (21).

RNA-RNA interactions in humans, including 66 lncRNA-mRNA interactions, 85 lncRNA-miRNA interactions and 8,368 miRNA-mRNA interactions, were retrieved from RAID v.2.0 with a confidence score of $\geq 0.8$ (18).

Classification of brain regions using a gene pair-based method. Gene pairs exhibiting correlated expression changes are likely to collectively perform a common function rather than such a function being performed by a single gene. Therefore, gene pairs were predicted to be more strongly associated with AD compared with genes functioning alone. A gene pair-based method was established to classify brain regions into different regional groups according to how each region is affected by AD.

Firstly, a $\mathrm{C}(2, \mathrm{~N}) \times \mathrm{M}$ correlation coefficient matrix was calculated followed by a Spearman's rank correlation coefficient, $r_{n m}$, calculation for each pair of $\mathrm{N}$ genes in each of the $\mathrm{M}$ regions (Fig. 1B).

$$
r_{n m}=\rho_{g_{X m}, g_{Y m}}=\frac{\operatorname{cov}\left(g_{X m}, g_{Y m}\right)}{\sigma_{g_{X m}} \sigma_{g_{Y m}}}
$$

$\mathrm{n}$ and $\mathrm{m}$ refers to the row and column in the matrix, respectively; $\mathrm{g}_{\mathrm{Xm}}$ and $\mathrm{g}_{\mathrm{Ym}}$ refers to the sample rank of gene $\mathrm{X}$ and gene $\mathrm{Y}$ in the region, respectively; $\rho$ represents the rank-based converted Pearson correlation coefficient; cov and $\sigma$ represent the covariance and the standard deviations of the rank variables, respectively; and $\mathrm{C}(2, \mathrm{~N})$ refers to the 2-combinations of an $\mathrm{N}$-element set.

Subsequently, the difference between each of the two brain regions for each gene pair was determined via Spearman's rank correlation coefficients and the sample size for each region was determined using the $\mathrm{R} /$ Bioconductor package cocor v 1.1-3 (22) using a $\mathrm{C}(2, \mathrm{~N}) \times \mathrm{M}^{2}$ matrix calculation. Each gene pair was scored using a sum-normalized vote strategy, which is the votes divided by the sum, to determine the differential expression of correlated gene pairs across regions.

Finally, PCA was used to calculate the contribution score for each differentially expressed gene pair. Principal component 1 (PC1) and principal component 2 (PC2) were used to classify brain regions into different groups.

$n c R N A$-mediated gene regulatory module identifications. A network was constructed using gene pairs that were revealed to exhibit the top $1 \%$ contribution rate in classified brain regions following $\mathrm{PC} 1$ and $\mathrm{PC} 2$ analyses. MCODE v 1.4.2 (23), a Cytoscape (24) plugin, was used to determine the functional modules that represented highly interconnected clusters of a network. Identified modules were integrated with 8,519 RNA-RNA interactions using RAID v 2.0 (18) with a confidence score of $\geq 0.8$ in order to reveal a ncRNA-mediated gene regulatory module (Fig. 1C).

Kyoto Encyclopedia of Genes and Genomes (KEGG) enrichment analysis. The enrichment of KEGG (25-27) pathways was performed using Database for Annotation, Visualization and Integrated Discovery (DAVID) v 6.8 (28). Significantly enriched pathways were defined as $\mathrm{P}<0.05$.

Heatmap analysis. Heatmap, representing data values using a color-coding system, was generated using the R/Bioconductor package gplots $\mathrm{v} 3.0 .1$ (29).

\section{Results}

Batch effects induced by technical variance are removed. Following the collection of gene expression data from HG-U133A, HG-U133B, and HG-U133_Plus_2 platforms, it 

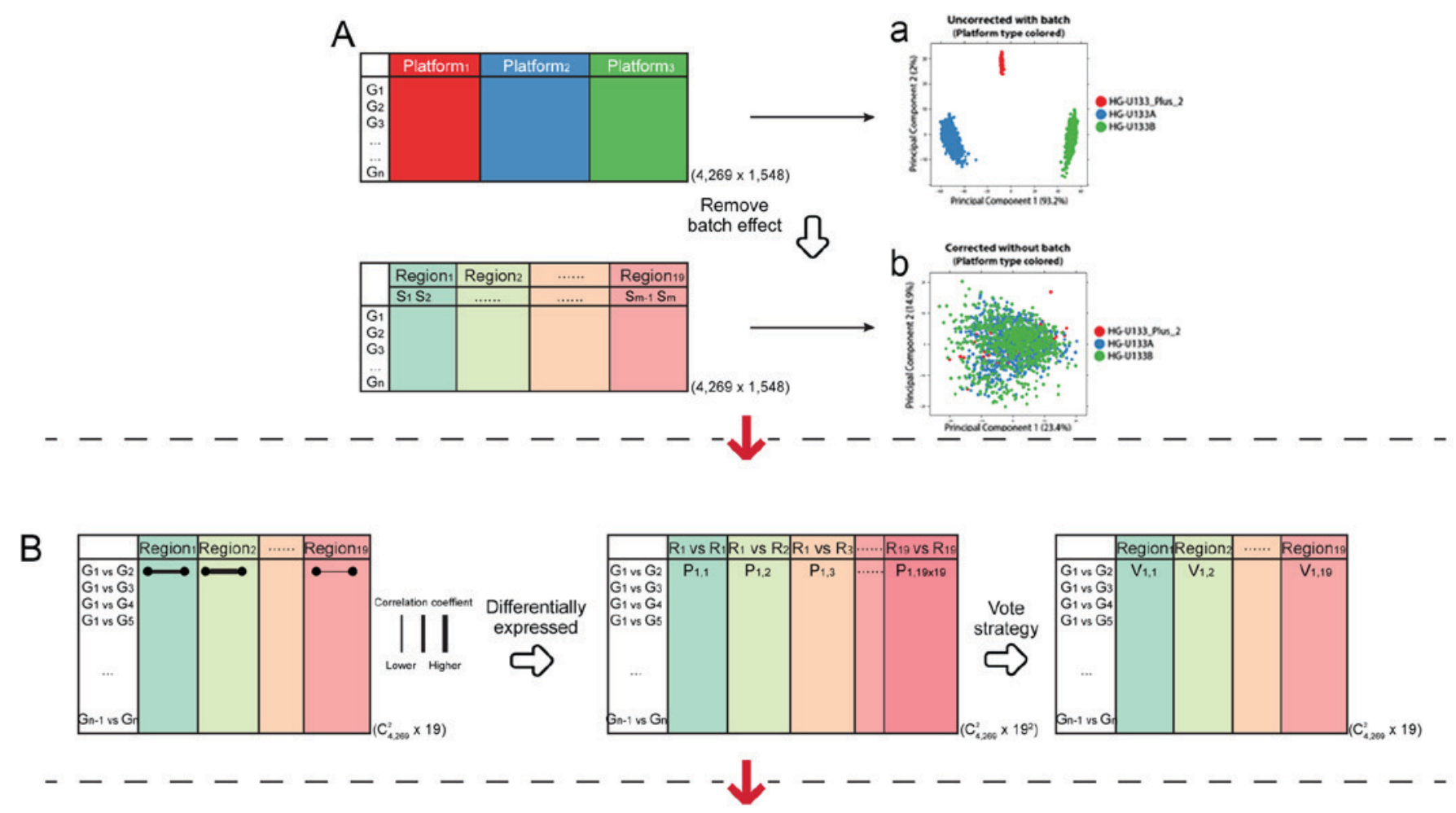

C

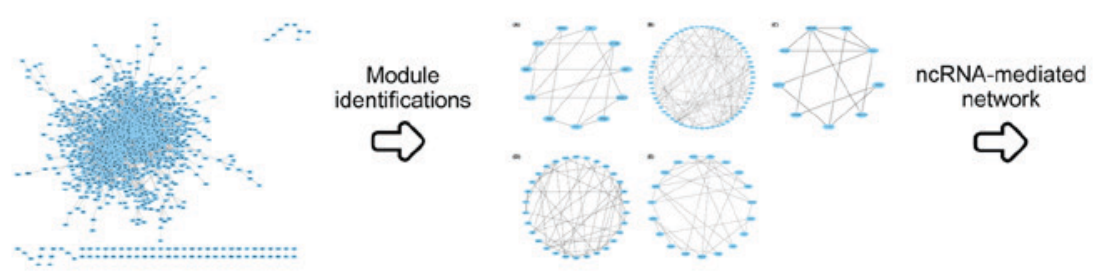

Nervous system-related ncRNA-mediated

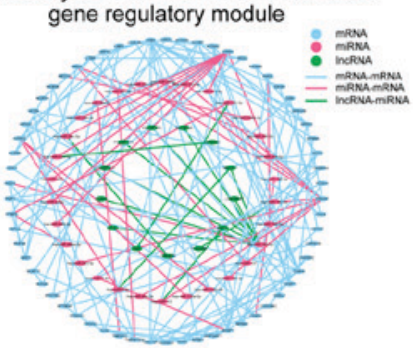

Figure 1. Flow chart of modularity identification based on a ncRNA-mediated gene pair-based regulatory network in Alzheimer's disease. (A) Removal of batch effects introduced by technical variance. (Aa-b) Schematic diagram of principal component analysis showing the classification of samples. (B) Gene pair-based method used to classify 19 brain regions. (C) Module identifications integrated with an ncRNA-mediated network in order to determine the ncRNA-mediated gene-pair-based regulatory network. ncRNA, non-coding RNA.

was revealed that there were marked differences in the top 500 genes based on the variance of gene expression between the three platforms in all samples; however, no marked differences were demonstrated between different brain regions (Fig. 2A). Following the removal of the batch effect by the $\mathrm{R} /$ Bioconductor package $s v a \vee 3.24 .4$ (20), a satisfactory profile was obtained when samples did not exhibit any marked differences based on their generated platform (Fig. 2B).

Gene pair-based method is used to classify 19 brain regions. Based on the established gene pair-based method, seven region groups were identified, including six out of 19 brain regions (31.6\%) exhibiting different patterns of expressed gene pairs from each other, and an additional 13 clustered regions exhibiting different patterns from the six regions as presented in Fig. 3A, which suggested that despite the 19 brain regions being situated physically adjacent to one another, numerous regions, particularly the amygdala, caudate nucleus, nucleus accumbens, precentral gyrus, putamen and temporal pole regions, exhibited different expression levels of gene pairs. In order to investigate the mechanisms underlying the differential expression of gene-pairs in the identified brain regions, 4,731 gene-pairs (including 1,308 genes) that represented the top $1 \%$ contribution in PC1 and PC2 (Fig. 3B) were used to classify the different brain regions. Annotation enrichment analysis of these 1,308 genes using DAVID (28) demonstrated that 63 KEGG (25-27) pathways were significantly annotated $(\mathrm{P}<0.05)$, the top 10 pathways of which are presented in Fig. 3C. The top five pathways, including dopaminergic synapse, retrograde endocannabinoid signaling, circadian entrainment, morphine addiction and glutamatergic synapse, demonstrated a strong association between the 1,308 genes and pathways associated with the nervous system. For example, the majority of genes enriched in the dopaminergic synapse pathway were regulated by the same genes (for example protein kinase A) or regulated each other, highlighting the importance of these enriched genes in this pathway.

The ncRNA-mediated gene regulatory module is identified. To further analyze the functions and cellular processes 
A

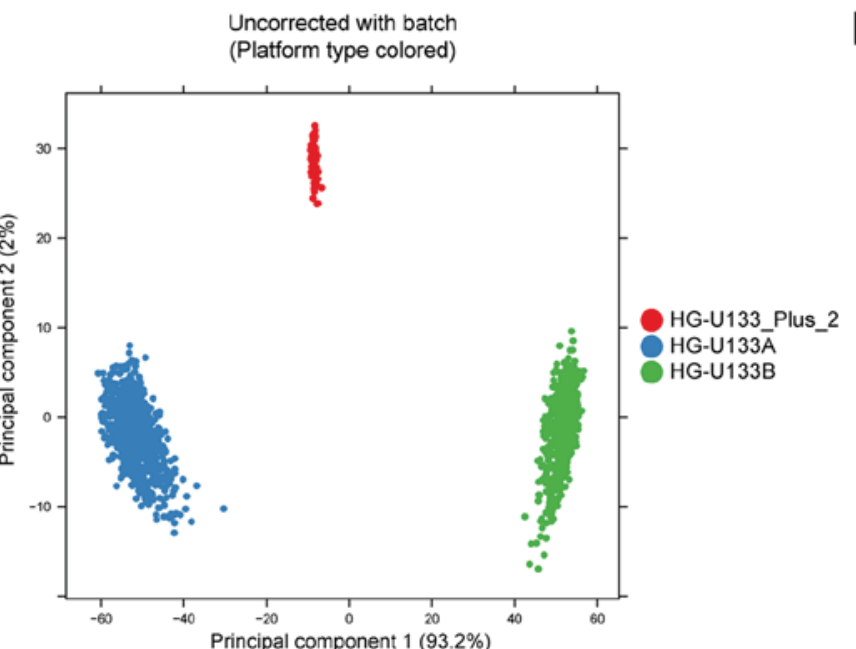

B

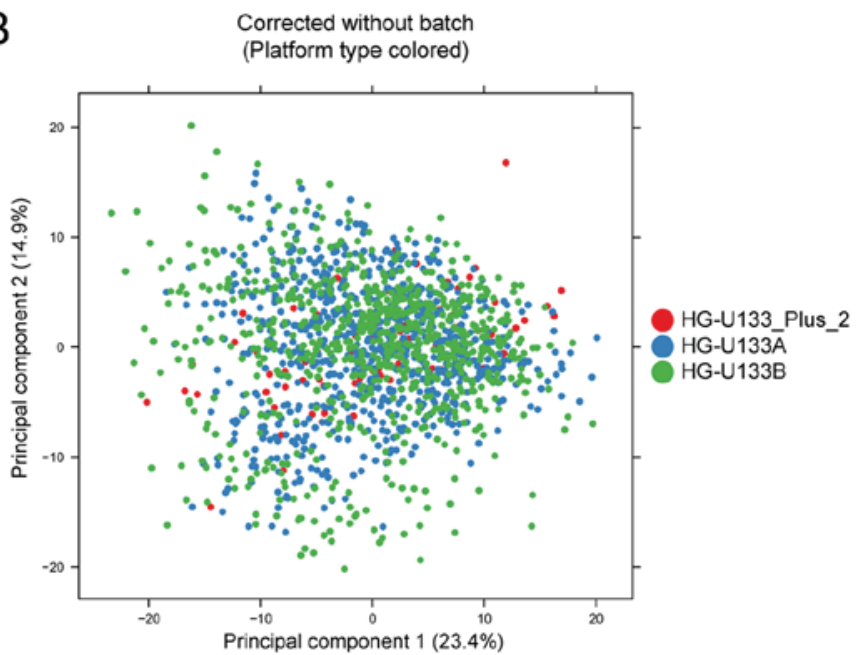

Figure 2. (A) Separation of gene pairs obtained from HG-U133A, HG-U133B and HG-U133_Plus_2 platforms via principal component analysis before removal of batch effects. (B) The differences between the platforms were corrected following removal of batch effect.

A

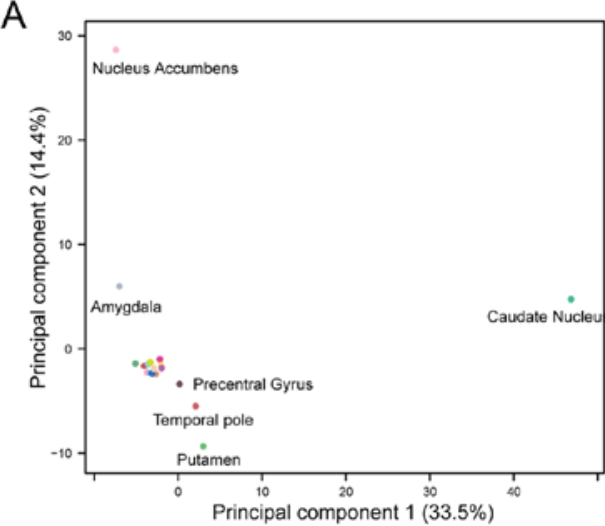

C

KEGG pathway enrichment analysis of differentially expressed gene pairs
B
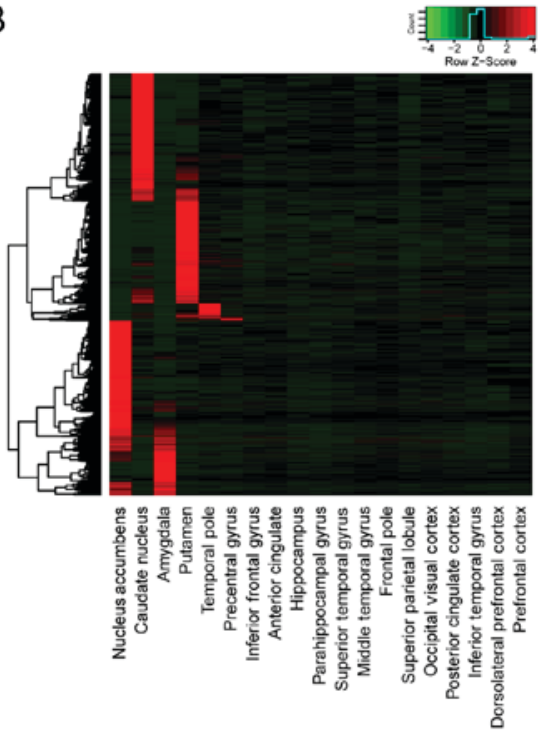
Retrograde endocannabinoid signaling Circadian entrainment Morphine addiction

Glutamatergic synapse cAMP signaling pathway Cholinergic synapse Choline metabolism in cancer Long-term depression GABAergic synapse

Figure 3. (A) Separation of 6 brain regions and one 13-region cluster via principal component analysis using the gene pair-based method. (B) Heatmap of the sum-normalized vote strategy scores of 4,731 gene pairs, which were represented in the top $1 \%$ contribution in principal component 1 and principal component 2 analyses. Rows and columns represent gene pairs and brain regions, respectively. (C) The top 10 significantly enriched KEGG pathways (P<0.05) of 4,731 differentially expressed gene-pairs (including 1,308 genes) in the identified brain regions. KEGG, Kyoto Encylopedia of Genes and Genomes.

the 4,731 gene pairs are involved in, these were used to construct an interaction network involving 1,308 genes and 4,731 interactions. Using a Cytoscape plugin $\operatorname{MCODE}(23,24)$, 5 highly interconnected modules were identified (Fig. 4A-E). Enrichment analysis of functional Module 2 and Module 3 demonstrated a strong correlation between pathways associated with the nervous system, including cholinergic synapses $(\mathrm{P}=0.04)$, circadian entrainment $(\mathrm{P}=0.01)$ and dopaminergic synapse $(\mathrm{P}=0.02)$. Notably, Module 2 was revealed to exhibit numerous interactions with miRNAs, which were revealed via integration with the ncRNA-mediated network to interact with a number of lncRNAs to form a ncRNA-mediated gene regulatory module, involving 105 molecules and 144 interactions (Fig. 4F). For example, surplus neurotrophic receptor tyrosine kinase (NTRK3) and peptidyl arginine deiminase 1 (PADIl) genes were 
A

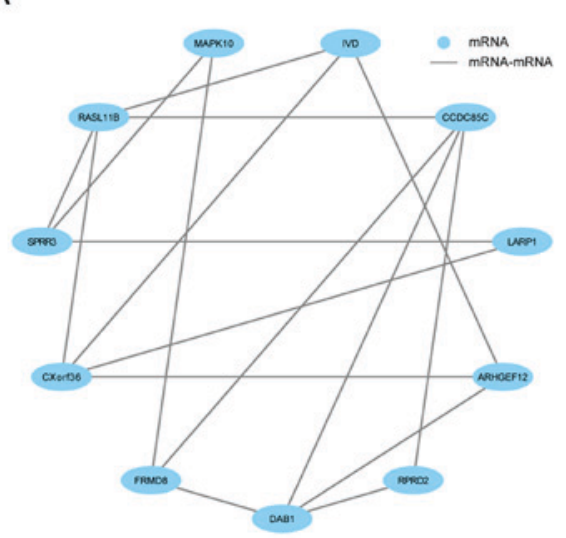

C

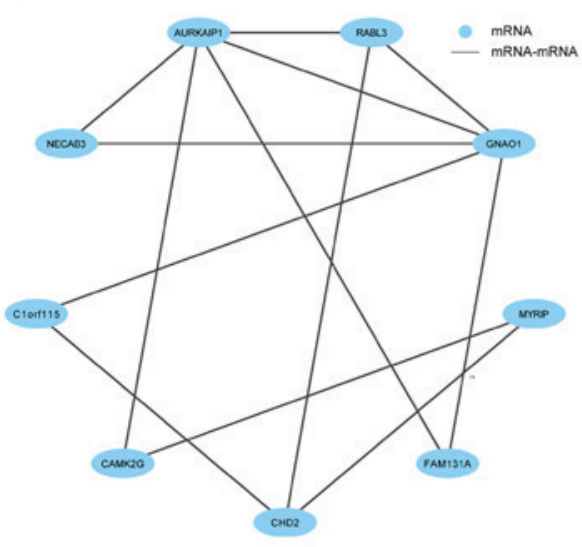

B

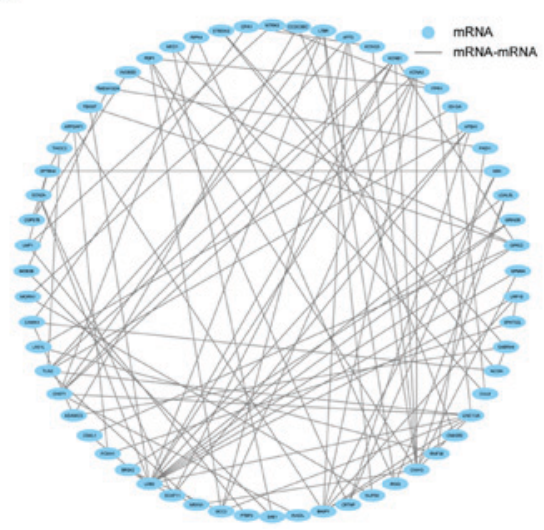

D

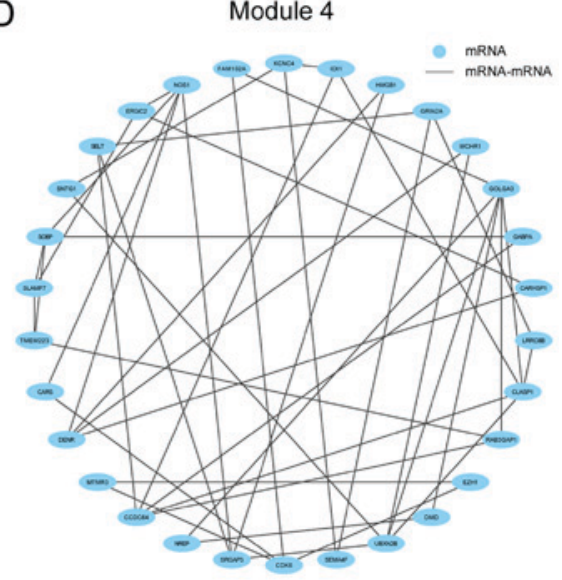

F Nervous system-related ncRNA-mediated gene regulatory module

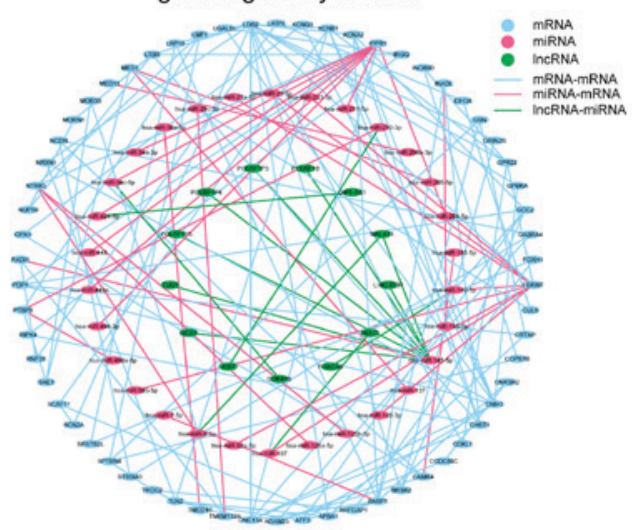

Figure 4. (A) Module 1, (B) Module 2, (C) Module 3, (D) Module 4 and (E) Module 5 are five highly interconnected modules detected by the network constructed using the gene pair-based method. The nodes and the edges indicated the mRNAs and the interactions between them. (F) A ncRNA-mediated gene regulatory module associated with the nervous system was established. The nodes in blue, red and green represent mRNAs, miRNAs and lncRNAs, respectively. The edges in blue, red and green represent interactions between mRNAs, miRNAs and mRNAs, and lncRNAs and miRNAs, respectively. mRNA, messenger RNA; miRNA, microRNA; ncRNA, non-conding RNA; lncRNA, long non-coding RNA.

demonstrated to interact with surplus miRNAs, including hsa-miR-9-5p, hsa-miR-125a-5p, hsa-miR-125b-5p and $h s a-m i R-145-5 p$; which were also revealed to interact with surplus lncRNAs, including long intergenic non-protein coding RNA, regulator of reprogramming; MALAT1, POU class 5 homeobox (POU5F)1B, POU5F1 pseudogene (P)3, POU5F1P4, POU5F1P5, TUG1 and urothelial cancer associated $1(U C A l)$. This suggested that these genes and lncRNAs may act as competing endogenous (ce)RNAs, by competing for binding of miRNAs. Many of the predicted ceRNAs have been previously reported as genes and lncRNAs associated with AD, including NTRK3, PADI1, MALAT1, POU5F1B, POU5F1P4, TUG1 and UCA1 (30-35). Therefore, the results suggested that 1ncRNAs, including $P O U 5 F 1 P 3$ and $P O U 5 F 1 P 5$, may perform similar functions related to the nervous system like $P O U 5 F 1 B$ and $P O U 5 F 1 P 4$, which have previously been demonstrated to be associated with AD. In conclusion, this indicated that the ncRNA-mediated 
gene regulatory module may aid the understanding of the mechanisms underlying $\mathrm{AD}$, as well as the development of novel therapeutic treatment strategies for patients with AD.

\section{Discussion}

AD represents the most common neurological disorder worldwide, and has been previously reported to differentially affect various brain regions $(3,4)$. The aim of the present study was to identify five functional modules of brain regions associated with AD in 90 patients, which may be utilized to classify 19 brain regions into seven groups, including marked disparate groupings of six single regions and a cluster of another 13 regions using a gene pair-based method. Following this, five modules were used to further analyze associations with ncRNAs using the ncRNA-mediated network. Finally, genes involved in one of the ncRNA-mediated gene regulatory modules identified by the present study were revealed to be significantly enriched in pathways associated with the nervous system, such as the cholinergic synapse pathway. In addition, in agreement with previous studies that revealed that NTRK 3 and PADII genes, as well as MALATI and TUGI lncRNAs in this module were associated with AD (30-35), it can be inferred that POU5FIP3 and POU5F1P5 lncRNAs compete for binding a pool of miRNAs with $N T R K 3$ and PADIl genes, which provides novel insight into the mechanisms underlying AD.

Unlike traditional biomarker identification that identifies single molecules, the gene pair-based method established in the present study, which integrated the ncRNA-mediated gene regulatory network, may better represent the complex mechanisms underlying $\mathrm{AD}$, as it includes associations between lncRNAs, miRNAs and mRNAs. For example, in the ncRNA-mediated gene regulatory module identified in the present study, hepatocellular carcinoma upregulated lncRNA and forkhead box O3B pseudogene lncRNAs were revealed to compete for binding with surplus miRNAs, such as $h s a-m i R-182-5 p$ and $h s a-m i R-107$. Furthermore, opa interacting protein 5-antisense RNA 1 and cyclin dependent kinase 4 pseudogene 1 lncRNAs as well as the inositol 1,4,5-trisphosphate receptor type 1 gene were revealed to compete for binding with miRNAs, such as $h s a-m i R-424-5 p$ and $h s a-m i R-34 c-5 p$. Therefore, such ceRNAs and miRNAs may represent novel candidate biomarkers for AD.

In conclusion, the present study established a gene pair-based method incorporated with ncRNA-mediated gene regulatory networks to identify module biomarkers associated with different brain regions, including lncRNAs, miRNAs and mRNAs. The results of the present study suggested that in order to comprehensively develop biomarker prediction methods and therapeutic strategies for the treatment of patients with $\mathrm{AD}$, the interactions among cellular molecules must be determined.

\section{Acknowledgements}

Not applicable.

\section{Funding}

No funding was received.

\section{Availability of data and materials}

All data generated or analyzed during this study are included in this published article.

\section{Authors' contributions}

LY and SW conceived and designed the experiments. LY and SQ analyzed the data. LY and SW wrote the manuscript. All authors read and approved the final manuscript.

\section{Ethics approval and consent to participate}

Not applicable.

\section{Patient consent for publication}

Not applicable.

\section{Competing interests}

The authors declare that they have no competing interests.

\section{References}

1. Kumar A, Singh A and Ekavali: A review on Alzheimer's disease pathophysiology and its management: An update. Pharmacol Rep 67: 195-203, 2015.

2. Maiese K: Late onset Alzheimer's disease: Novel clinical prospects for the future. Curr Neurovasc Res 14: 89, 2017.

3. Wang M, Roussos P, McKenzie A, Zhou X, Kajiwara Y, Brennand KJ, De Luca GC, Crary JF, Casaccia P, Buxbaum JD, et al: Integrative network analysis of nineteen brain regions identifies molecular signatures and networks underlying selective regional vulnerability to Alzheimer's disease. Genome Med 8: 104, 2016.

4. Wang X, Michaelis ML and Michaelis EK: Functional genomics of brain aging and Alzheimer's disease: Focus on selective neuronal vulnerability. Curr Genomics 11: 618-633, 2010.

5. Collins FS, Morgan M and Patrinos A: The Human Genome Project: Lessons from large-scale biology. Science 300: 286-290, 2003.

6. Constantinides VC, Paraskevas GP, Emmanouilidou E, Petropoulou O, Bougea A, Vekrellis K, Evdokimidis I, Stamboulis E and Kapaki E: CSF biomarkers $\beta$-amyloid, tau proteins and a-synuclein in the differential diagnosis of Parkinson-plus syndromes. J Neurol Sci 382: 91-95, 2017.

7. Lin PP, Chen WL, Yuan F, Sheng L, Wu YJ, Zhang WW, Li GQ, Xu HR and Li XN: An UHPLC-MS/MS method for simultaneous quantification of human amyloid beta peptides $A \beta 1-38$, $\mathrm{A} \beta 1-40$ and $\mathrm{A} \beta 1-42$ in cerebrospinal fluid using micro-elution solid phase extraction. J Chromatogr B Analyt Technol Biomed Life Sci 1070: 82-91, 2017.

8. Chopra P, Lee J, Kang J and Lee S: Improving cancer classification accuracy using gene pairs. PLoS One 5: e14305, 2010.

9. Jin N, Wu H, Miao Z, Huang Y, Hu Y, Bi X, Wu D, Qian K, Wang L, Wang C, et al: Network-based survival-associated module biomarker and its crosstalk with cell death genes in ovarian cancer. Sci Rep 5: 11566, 2015.

10. Knoll M, Lodish HF and Sun L: Long non-coding RNAs as regulators of the endocrine system. Nat Rev Endocrinol 11: 151-160, 2015.

11. ENCODE Project Consortium,Birney E,Stamatoyannopoulos JA, Dutta A, Guigó R, Gingeras TR, Margulies EH, Weng Z, Snyder M, Dermitzakis ET, et al: Identification and analysis of functional elements in $1 \%$ of the human genome by the ENCODE pilot project. Nature 447: 799-816, 2007.

12. Bertone P, Stolc V, Royce TE, Rozowsky JS, Urban AE, Zhu X, Rinn JL, Tongprasit W, Samanta M, Weissman S, et al: Global identification of human transcribed sequences with genome tiling arrays. Science 306: 2242-2246, 2004. 
13. Wang $\mathrm{KC}$ and Chang $\mathrm{HY}$ : Molecular mechanisms of long noncoding RNAs. Mol Cell 43: 904-914, 2011.

14. Sheinerman KS, Toledo JB, Tsivinsky VG, Irwin D, Grossman M, Weintraub D, Hurtig HI, Chen-Plotkin A, Wolk DA, McCluskey LF, et al: Circulating brain-enriched microRNAs as novel biomarkers for detection and differentiation of neurodegenerative diseases. Alzheimers Res Ther 9: 89, 2017.

15. Cesana M, Cacchiarelli D, Legnini I, Santini T, Sthandier O, Chinappi M, Tramontano A and Bozzoni I: A long noncoding RNA controls muscle differentiation by functioning as a competing endogenous RNA. Cell 147: 358-369, 2011

16. Tay Y, Kats L, Salmena L, Weiss D, Tan SM, Ala U, Karreth F, Poliseno L, Provero P, Di Cunto F, et al: Coding-independent regulation of the tumor suppressor PTEN by competing endogenous mRNAs. Cell 147: 344-357, 2011.

17. Cai Y, Sun Z, Jia H, Luo H, Ye X, Wu Q, Xiong Y, Zhang W and Wan J: Rpph1 upregulates CDC42 expression and promotes hippocampal neuron dendritic spine formation by competing with miR-330-5p. Front Mol Neurosci 10: 27, 2017.

18. Yi Y, Zhao Y, Li C, Zhang L, Huang H, Li Y, Liu L, Hou P, Cui T, Tan P, et al: RAID v2.0: An updated resource of RNA-associated interactions across organisms. Nucleic Acids Res 45 (D1): D115-D118, 2017.

19. Irizarry RA, Hobbs B, Collin F, Beazer-Barclay YD, Antonellis KJ, Scherf U and Speed TP: Exploration, normalization, and summaries of high density oligonucleotide array probe level data. Biostatistics 4: 249-264, 2003.

20. Leek JT, Johnson WE, Parker HS, Jaffe AE and Storey JD: The sva package for removing batch effects and other unwanted variation in high-throughput experiments. Bioinformatics 28 $882-883,2012$

21. Leek JT and Storey JD: Capturing heterogeneity in gene expression studies by surrogate variable analysis. PLoS Genet 3: $1724-1735,2007$

22. Diedenhofen B and Musch J: Cocor: A comprehensive solution for the statistical comparison of correlations. PLoS One 10: e0121945, 2015.

23. Bader GD and Hogue CW: An automated method for finding molecular complexes in large protein interaction networks. BMC Bioinformatics 4: 2, 2003.

24. Shannon P, Markiel A, Ozier O, Baliga NS, Wang JT, Ramage D, Amin N, Schwikowski B and Ideker T: Cytoscape: A software environment for integrated models of biomolecular interaction networks. Genome Res 13: 2498-2504, 2003.
25. Kanehisa M, Furumichi M, Tanabe M, Sato Y and Morishima K: KEGG: New perspectives on genomes, pathways, diseases and drugs. Nucleic Acids Res 45: D353-D361, 2017.

26. Kanehisa M, Sato Y, Kawashima M, Furumichi M and Tanabe M KEGG as a reference resource for gene and protein annotation. Nucleic Acids Res 44 (D1): D457-D462, 2016.

27. Kanehisa M and Goto S: KEGG: Kyoto encyclopedia of genes and genomes. Nucleic Acids Res 28: 27-30, 2000.

28. Dennis G Jr, Sherman BT, Hosack DA, Yang J, Gao W, Lane HC and Lempicki RA: DAVID: Database for annotation, visualization, and integrated discovery. Genome Biol 4: P3, 2003.

29. Warnes G, Bolker B, Bonebakker L, Gentleman R, Andy Liaw WH, Lumley T, Maechler M, Magnusson A, Moeller S, Schwartz $M$ and Venables B: gplots: Various $\mathrm{R}$ programming tools for plotting data. $\mathrm{R}$ package version 3.0.3, 2016.

30. Braskie MN, Kohannim O, Jahanshad N, Chiang MC, Barysheva M, Toga AW, Ringman JM, Montgomery GW, McMahon KL, de Zubicaray GI, et al: Relation between variants in the neurotrophin receptor gene, NTRK3, and white matter integrity in healthy young adults. NeuroImage 82: 146-153, 2013.

31. Chavanas S, Adoue V, Méchin MC, Ying S, Dong S, Duplan H, Charveron M, Takahara H, Serre G and Simon M: Long-range enhancer associated with chromatin looping allows AP-1 regulation of the peptidylarginine deiminase 3 gene in differentiated keratinocyte. PLoS One 3: e3408, 2008.

32. Yao J, Wang XQ, Li YJ, Shan K, Yang H, Wang YN, Yao MD, Liu C, Li XM, Shen Y, et al: Long non-coding RNA MALAT1 regulates retinal neurodegeneration through CREB signaling. EMBO Mol Med 8: 346-362, 2016.

33. Costa V, Esposito R, Aprile M and Ciccodicola A: Non-coding RNA and pseudogenes in neurodegenerative diseases: 'The (un) Usual suspects'. Front Genet 3: 231, 2012.

34. Johnson R: Long non-coding RNAs in Huntington's disease neurodegeneration. Neurobiol Dis 46: 245-254, 2012.

35. Zheng J, Yi D, Liu Y, Wang M, Zhu Y and Shi H: Long nonding RNA UCA1 regulates neural stem cell differentiation by controlling miR-1/Hes1 expression. Am J Transl Res 9: 3696-3704, 2017.

This work is licensed under a Creative Commons Attribution-NonCommercial-NoDerivatives 4.0 International (CC BY-NC-ND 4.0) License. 